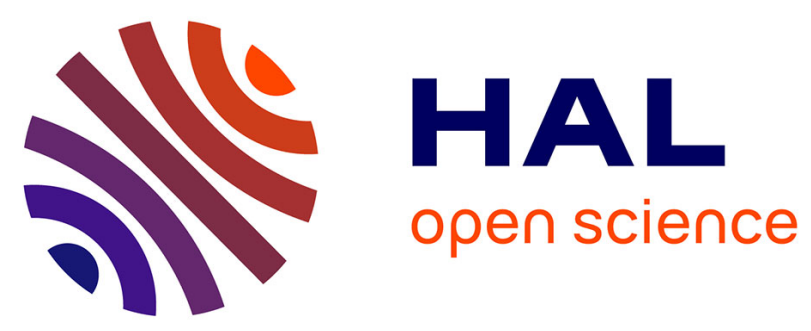

\title{
Synthesis process and thermoelectric properties of n-type tin selenide thin films
}

\author{
W.-T. Wang, Z.-H. Zheng, F. Li, C. Li, P. Fan, J.-T. Luo, B. Li
}

\section{To cite this version:}

W.-T. Wang, Z.-H. Zheng, F. Li, C. Li, P. Fan, et al.. Synthesis process and thermoelectric properties of n-type tin selenide thin films. Journal of Alloys and Compounds, 2018, 763, pp.960-965. 10.1016/j.jallcom.2018.06.021 . hal-01835022

HAL Id: hal-01835022

https://hal-univ-rennes1.archives-ouvertes.fr/hal-01835022

Submitted on 13 Jul 2018

HAL is a multi-disciplinary open access archive for the deposit and dissemination of scientific research documents, whether they are published or not. The documents may come from teaching and research institutions in France or abroad, or from public or private research centers.
L'archive ouverte pluridisciplinaire HAL, est destinée au dépôt et à la diffusion de documents scientifiques de niveau recherche, publiés ou non, émanant des établissements d'enseignement et de recherche français ou étrangers, des laboratoires publics ou privés. 


\section{Synthesis Process and Thermoelectric Properties of n-type Tin}

\section{Selenide Thin Films}

Wen-ting Wang ${ }^{\mathrm{a}}$, Zhuang-hao Zheng ${ }^{\mathrm{a}, \mathrm{b}}, \mathrm{Fu} \mathrm{Li}^{\mathrm{a}}{ }^{*}, \mathrm{Cao} \mathrm{Li}^{\mathrm{a}}$, Ping Fan ${ }^{\mathrm{a}, *}$, Jing-ting Luo ${ }^{\mathrm{a}}, \mathrm{Bo} \mathrm{Li}^{\mathrm{c}}$,

${ }^{a}$ Shenzhen Key Laboratory of Advanced Thin Films and Applications, College of Physics and Energy, Shenzhen University 518060, China.

${ }^{\mathrm{b}}$ Laboratory of Glasses and Ceramics, Institute of Chemical Science UMR CNRS 6226, University of Rennes 1, Rennes 35042, France.

${ }^{c}$ Advanced Materials Institute, Graduate School at Shenzhen, Tsinghua University, Shenzhen 518055, China.

*Corresponding authors. Email: lifu@szu.edu.cn and fanping@szu.edu.cn

\section{Abstract:}

Tin selenide $(\mathrm{SnSe})$ is a very promising thermoelectric material, but there are few reports related to the thermoelectric properties of its thin films, especially for n-type SnSe. In this work, n-type SnSe thin films were synthesized via thermal evaporation using powdered SnSe that was prepared directly by mechanical alloying. It was found that the crystallinity of the films gradually improved and the closer the elemental ratio of $\mathrm{Sn}$ and $\mathrm{Se}$ was to a ratio of 1:1 with increasing the current through the tungsten boat during the thermal evaporation of the SnSe precursor from 100 to 130 A. Further, the thin films obtained at 120 A show the highest power factor $\left(0.13 \mu \mathrm{W} / \mathrm{mK}^{2}\right)$ of all the samples fabricated in this study. The samples were then annealed, and the thermoelectric properties of the films were enhanced as this step improved their crystallinities. A Seebeck coefficient of over $100 \mu \mathrm{V} / \mathrm{K}$ could be achieved with an annealing time of $4 \mathrm{~h}$. Combined with a recorded electrical conductivity of $47 \mathrm{~S} / \mathrm{cm}$, a maximum power factor of $120 \mu \mathrm{W} / \mathrm{mK}^{2}$ at $473 \mathrm{~K}$ has been obtained for the annealed film with n-type conductive characteristics; this is nearly six times higher than that reported for polycrystalline n-type bulk $\mathrm{SnSe}$ at the same temperature.

Key words: tin selenide; thermoelectric property; thermal evaporation 


\section{Introduction}

Thermoelectric (TE) materials are attracting increasing attention owing to their promising applications in waste heat recovery and electronic cooling [1-3]. The performance of TE materials can be evaluated by the dimensionless figure of merit, $Z T=S^{2} \sigma T / \kappa$, where $S$ is the Seebeck coefficient, $\sigma$ is the electrical conductivity, $T$ is the absolute temperature, and $\kappa$ is the thermal conductivity. A larger ZT value means a higher TE conversion efficiency of the corresponding device. Thus, to obtain a good device, excellent electrical transport properties, along with a low thermal conductivity to achieve a high $Z T$ for the material, are required. As there is a constraint relationship between the electrical and thermal transport properties, the search for practical materials with large $Z T$ values is challenging. Thin films with two dimensional structures have been received significant attention in the field of TE materials, because their thermal and electrical transport properties can be controlled independently [4]. In addition, flexible generators made using thin films have considerable potential for applications in the microelectronics industry [5,6]. Therefore, research into preparing high performance thin films has become important within the field of thermoelectricity [7].

Tin selenide ( $\mathrm{SnSe}$ ) crystallized with a layered crystal structure is known to be a narrow band gap semiconductor with an indirect band gap of $0.90 \mathrm{eV}$ at room temperature and direct band gap of $1.30 \mathrm{eV}$ at high temperatures $(\sim 750 \mathrm{~K})$ [8]. It has been intensively investigated already as it is an earth abundant and environmentally benign component of photovoltaic devices such as thin film solar cells, where it is used because its band gap falls within the optimum range for photovoltaic applications $[9,10]$. Recently, SnSe has been investigated for its suitability for TE applications because of its environmentally friendly composition and the high ZT value of single crystalline $\mathrm{SnSe}$, which is attributed to its extremely low thermal conductivity and moderate electrical transport properties [11,12]. However, the layered crystal structure causes the poor mechanical properties of the single crystalline SnSe, since it can easily cleave along the (001) plane [13]. Therefore, researchers have focused their attentions on polycrystalline SnSe owing to its good 
mechanical properties and easily controlled production conditions. To date, most studies on polycrystalline SnSe have focused on p-type SnSe, with only few reports related to n-type one $[14,15]$. In particular, reports on the TE properties of n-type SnSe thin films are scarce.

In this work, n-type SnSe thin films were prepared by thermal evaporation. The precursor for the thermal evaporation step was synthesized by mechanical alloying using commercial Sn and Se powders; this is a simple and cost-effective approach for fabricating high-performance TE materials that has emerged in recent years $[16,17]$. The physical properties of thin films are extremely sensitive to the growth conditions and the type of deposition technique employed for their growth. Thus, we investigated the composition, microstructure, and TE properties with an emphasis on the effect of the synthesis conditions, including the applied current to the crucible holding the SnSe precursor during thermal evaporation as well as the annealing temperature.

\section{Experimental details}

SnSe thin films were prepared by thermal evaporation using powders that were directly prepared by mechanical alloying with commercial Sn (99.999\%) and Se (99.999\%) powders as the raw materials according to the nominal composition of SnSe. The thin films were fabricated on $20 \mathrm{~mm} \times 20 \mathrm{~mm} \times 2 \mathrm{~mm} \mathrm{BK7}$ glass substrates under $4 \times 10^{-3} \mathrm{~Pa}$ by using a FJL-520 ultra-high vacuum system with different current of 100,110, 120, and 130 A on tungsten boat. The substrate glass slides were cleaned using air-laid paper after being cleaned in an ultrasonic bath of (sequentially) acetone, alcohol, and deionized water for $20 \mathrm{~min}$ each prior to thermal evaporation. After the thermal evaporation step, the films were annealed at 473, 573, and $673 \mathrm{~K}$ for $1 \mathrm{~h}$ under an argon atmosphere at $440 \mathrm{~Pa}$.

The phase structures of the SnSe thin films were probed using X-ray diffraction (XRD, Ultima IV, Rigaku, Japan) with the conventional $\theta-2 \theta$ mode. The microstructures were observed via scanning electron microscopy (SEM, Supra 55 Sapphire, Zeiss, Germany), and the composition was analyzed by energy dispersive spectroscopy (EDS). The thicknesses of all the films were measured by a Dektak3 ST 
surface-profile measurement system (Ultima4, Rigaku, Japan). The Seebeck coefficient and electrical conductivity were recorded simultaneously as a function of temperature (from 300 to $473 \mathrm{~K}$ ) using a Seebeck coefficient/electrical resistance measuring system (SBA458, Netzsch, Germany). The Hall mobility and Hall concentration were measured using a Hall effect measurement system (HL5500, Nanometric, USA). The uncertainty of Seebeck coefficient and electrical conductivity measurements is within 5\%. Therefore, the combined uncertainty for all measurements involved in power factor $\left(P F=S^{2} \sigma\right)$ determination shown in the plot is estimated to be $10 \%$. The standard deviation of the measured power factor below 450 $\mathrm{K}$ is within $5 \%$ for three different samples with the same composition and preparation technology.

\section{Results and discussion}

The phase structure and microstructure of SnSe powder prepared by mechanical alloying before thermal evaporation were firstly observed using XRD and SEM. The results indicated that all the locations of diffraction peaks of the powder corresponded well to those of the standard SnSe (JCPDS\#48-1224), indicating that a single, orthorhombic SnSe phase was synthesized. The synthesized powder, with nonuniform particle size of $100-500 \mathrm{~nm}$ in diameter, are aggregated to a certain extent. The average ratio of Sn to Se detected by EDS are close to the stoichiometry of SnSe. Fig. 1 shows the XRD patterns of unannealed SnSe thin films deposited at different crucible currents during the thermal evaporation. It shows that the samples are amorphous when the applied current is below $110 \mathrm{~A}$. As the current was increased, the as-deposited thin films started to show some characteristic diffraction peaks related to the (111), (311), and (411) plane of SnSe, indicating that the crystallinity of the films gradually enhanced. The SEM images of all samples are shown in Fig. 2. There are no obvious crystalline grains for the films deposited with currents of 100 and $110 \mathrm{~A}$. However, when the current was increased, more and more crystalline grains appear in the films [18]. The ratios of $\mathrm{Sn}$ to $\mathrm{Se}$ of the as-deposited films obtained under different currents were detected by EDS which are presented in Table 1. We found 
that the ratio of Sn to Se of all the films deviated from 1:1, although the ratio in the precursor was nearly $1: 1$. The content of $\mathrm{Sn}$ is obviously greater than that of Se. With increasing the applied current, the Se content increased and the ratio gradually started approaching 1:1. This means that the evaporation of Se is sensitive to the current during the thermal evaporation, which is likely caused by the different saturated vapor pressures of Sn and Se [10].

Fig. 3(a) shows the temperature dependence of the electrical conductivity for all unannealed films. The data show that the electrical conductivity increases along with the temperature above $350 \mathrm{~K}$ owing to thermal excitation, which indicates semiconducting behavior. With increasing temperature, the lattice is expanded and the orbital overlap between atoms is enhanced. This makes the energy band dispersion stronger, which consequently reduces the band gap. Thus, the smaller band-gap at a higher temperature made it easier for the carriers to be excited and thereby the carrier concentration would be increased. As a result, the electrical conductivity, which can be expressed as $\sigma=n e \mu$ (where $n$ is the carrier concentration, $\mu$ is the carrier mobility and $e$ is the electron charge), increases with increasing temperature. In addition, the electrical conductivities show a weak dependence on the thermal evaporation current, and the values for the films obtained at different conditions do not differ significantly. Around room temperature, the electrical conductivities are about $0.25 \mathrm{~S} / \mathrm{cm}$. When the temperature increases, the values rise to about $1.5 \mathrm{~S} / \mathrm{cm}$ at $450 \mathrm{~K}$. Compared with the reported results for bulk polycrystalline samples, the electrical conductivities of our films are lower, which is mainly owing to their lower carrier concentrations and mobility [19]. In fact, the Hall carrier concentration and mobility at room temperature of a representative film obtained at 130 A were measured to determine its transport properties. The results indicate that the carrier concentration was $2.56 \times 10^{17} \mathrm{~cm}^{-3}$, and the carrier mobility was only $2.80 \mathrm{~cm}^{2} / \mathrm{Vs}$ which is nearly one order of magnitude lower than that reported for a comparative bulk sample [20]. As discussed above, the crystallinity of that film sample appears to be poor. Therefore, we attribute the lower carrier concentration and mobility to the 
poor crystallinity.

The temperature dependence of the Seebeck coefficient of the fabricated films is shown in Fig. 3(b). It can be seen that all the values are negative, which indicates that these samples are n-type conductors with electrons as the dominate carriers. As mentioned above, the ratio of Sn to Se of all the films deviated from 1:1, and the films exhibited a lack of Se. Thus, Se vacancies will introduce more electrons, leading to an n-type conductive mechanism. This result is in agreement with a recent report in which a bulk sample with extra Sn was determined to be n-type with a negative Seebeck coefficient [21]. Moreover, similar to the electrical conductivity results as mentioned above, the Seebeck coefficient also shows a weak dependence on the applied current. As shown in Fig. 3(b), the absolute Seebeck coefficient for all the films roughly increased as the temperature was increased. Around room temperature, the absolute value was about $10 \mu \mathrm{V} / \mathrm{K}$; when the temperature increased to $450 \mathrm{~K}$, it improved to $27 \mu \mathrm{V} / \mathrm{K}$. However, because of poor crystallinity, all the values were still much lower than those reported for bulk samples [13]. Nevertheless, the Seebeck coefficient was greatly increased after annealing, which would be discussed below.

Combing the electrical conductivity and Seebeck coefficient, the power factors of the unannealed films were calculated as shown in Fig. 3(c). It can be seen that the power factor increases as the temperature increases, and that it rises sharply from 350 to $425 \mathrm{~K}$. As a result, a slightly higher power factor of $0.13 \mu \mathrm{W} / \mathrm{mK}^{2}$ was obtained for the film prepared at 120 A (as-deposited) because of its slightly higher absolute Seebeck coefficient. As discussed above, although the crystallinity of the film was enhanced as the $\mathrm{Sn}:$ Se ratio approached 1:1 when the thermal evaporation current was increased from 100 to $130 \mathrm{~A}$, both the Seebeck coefficient and the electrical conductivity displayed weak dependence on the evaporation current. And the values of the electrical conductivity and Seebeck coefficient were relatively low because of the films' poor crystallinity. Therefore, to improve the TE properties, the films prepared at $130 \mathrm{~A}$ with the best crystallinity were annealed at 473, 573, and $673 \mathrm{~K}$, respectively.

Fig. 4 shows the XRD patterns of the annealed films. All the samples display 
three major diffraction peaks, which can be assigned to the (111), (311), and (411) planes of SnSe (JCPDS\#48-1224). It should also be noted that some characteristic diffraction peaks in the range of $2<$ theta $>=45^{\circ}$ to $70^{\circ}$ appeared in the films after annealing, indicating that the crystallinity had improved. The surface morphologies for the annealed films are shown in Fig. 5. The images show that the grains grew after annealing. The average grain sizes for the films annealed at 473, 573 and $673 \mathrm{~K}$ were approximately 27,50 and $71 \mathrm{~nm}$ in diameter respectively, which increased with increasing the annealing temperature. Furthermore, the porosity of the films increased along with the temperature. It is reported that thermal induced elements segregation and evaporation took place in the sample and lead to phase separation as well as formation of large voids in the materials [21]. After determining the composition by EDS, we found that the Sn content decreased with increasing the annealing temperature. Since the film before annealing was Sn-rich sample as mentioned above, thus, it is deduced that the Sn elements might segregate preferentially and evaporate during thermal treatment, resulting in the formation of voids and reduction of the density of the film. The Sn to Se ratio was nearly 1:1 when the annealing temperature was either 573 or $673 \mathrm{~K}$. Moreover, the thickness of the film without annealing is around $550 \mathrm{~nm}$. After the films were annealed at 473, 573 and $673 \mathrm{~K}$, they have gradually reduced to 520,500 and $100 \mathrm{~nm}$, respectively. Notably, the decrease in thickness for the film annealed at $673 \mathrm{~K}$ is very pronounced, indicating that the films volatilized dramatically. In fact, when the annealing temperature was raised to over $673 \mathrm{~K}$, no or a few products can be found on the glass substrates because of the volatilization, thus practically limiting the annealing temperature to $673 \mathrm{~K}$.

Fig. 6 shows the electrical transport properties as a function of temperature for the films after heat treatment. The electrical conductivity as shown in Fig. 6(a) increased after annealing, and generally enhanced along with the annealing temperature. After the film was annealed at $673 \mathrm{~K}$ for $1 \mathrm{~h}$, the electrical conductivity improved to $1.0 \mathrm{~S} / \mathrm{cm}$ at room temperature, and then increased to $5.0 \mathrm{~S} / \mathrm{cm}$ as the temperature increased to $450 \mathrm{~K}$, which is twice higher than that of the unannealed films. Notably, this conductivity is also much larger than that reported for n-type bulk 
polycrystalline $\mathrm{SnSe}_{0.8}$ at the same temperature [22]. This enhanced electrical conductivity might be caused by the increased carrier concentration and carrier mobility owing to the heat treatment (which causes a recrystallization), since the annealed films have a better crystallinity and larger grain sizes [23,24]. Actually, the measured carrier concentration and carrier mobility of the film annealed at $573 \mathrm{~K}$ at room temperature were $1.53 \times 10^{18} \mathrm{~cm}^{-3}$ and $3.72 \mathrm{~cm}^{2} / \mathrm{Vs}$, and those of the film annealed at $673 \mathrm{~K}$ were $1.69 \times 10^{18} \mathrm{~cm}^{-3}$ and $4.21 \mathrm{~cm}^{2} / \mathrm{Vs}$. These values are larger than those of the unannealed film, which were $2.56 \times 10^{17} \mathrm{~cm}^{-3}$ and $2.80 \mathrm{~cm}^{2} / \mathrm{Vs}$, respectively. Additionally, it also shows that both of the carrier concentration and carrier mobility for the sample annealed at $673 \mathrm{~K}$ are slightly higher than those of the sample annealed at $573 \mathrm{~K}$. According to the XRD and SEM as mentioned above, the annealed films have a better crystallinity and larger grain sizes, which means that the grain size has increased and some types of crystal defects gradually disappear during the annealing process. Thus, better crystallinity, larger grain size, fewer boundaries and defects would all result in the improvement of the carrier concentration and mobility because of weak scattering $[25,26]$.

The Seebeck coefficients of the films after annealing are shown in Fig. 6(b). All the values are negative, suggesting the samples are n-type conductors. The absolute Seebeck coefficients are significantly increased compared with that before annealing; this was particularly the case for the samples annealed at 573 and $673 \mathrm{~K}$. As discussed above, the absolute value for the unannealed film was below $27 \mu \mathrm{V} / \mathrm{K}$ across the entire measured temperature range. However, it has improved to $200-250 \mu \mathrm{V} / \mathrm{K}$ after the sample was annealed at over $573 \mathrm{~K}$, thus increasing the Seebeck coefficient by one order of magnitude compared to the unannealed film. Furthermore, the Seebeck coefficient displayed weak temperature dependence, which is similar to the behavior reported for n-type bulk polycrystalline $\mathrm{SnSe}_{0.8}$ [22]. Combined with the improved electrical conductivity of over $5 \mathrm{~S} / \mathrm{cm}$ as mentioned above, the power factor is significantly enhanced, as shown in Fig. 6(c). A maximum power factor of 21 $\mu \mathrm{W} / \mathrm{mK}^{2}$ at $450 \mathrm{~K}$ was achieved for the film that was annealed at $673 \mathrm{~K}$, which displays n-type characteristics. It is worth noting that the power factor in this work is 
comparable with that reported for n-type bulk polycrystalline $\mathrm{SnSe}_{0.8}$ [22].

These results indicate that annealing is efficient for improving the TE properties of SnSe thin films. Thus we decided to conduct an additional experiment and increase the annealing time further to $4 \mathrm{~h}$. We found that the electrical transport properties were further enhanced so that they were even comparable to those reported for p-type bulk polycrystalline $\mathrm{SnSe}$ at the same temperature [20]. As shown in Fig. 7, the electrical conductivity was around $25 \mathrm{~S} / \mathrm{cm}$ at room temperature. As we increased the measurement temperature to $473 \mathrm{~K}$, the conductivity increased further to $47 \mathrm{~S} / \mathrm{cm}$, which is nearly one order of magnitude greater than that of the film annealed for $1 \mathrm{~h}$. The measurement results for the carrier concentration and mobility indicate that the significant enhancement of the electrical conductivity can be attributed to both the increased carrier concentration and mobility. The value of the carrier concentration at room temperature is $8.09 \times 10^{18} \mathrm{~cm}^{3}$, while that of the mobility is $19.31 \mathrm{~cm}^{2} / \mathrm{Vs}$; these values are comparable to those reported for bulk samples. After observing the phase structure of the annealed films by XRD, it is found that a small amount of impure $\mathrm{SnSe}_{2}$ phase appeared after annealing the films for $4 \mathrm{~h}$. Thus, we propose that the enhanced carrier concentration is caused by the $\mathrm{SnSe}_{2}$ impurities, since $\mathrm{SnSe}_{2}$ is an n-type conductor [3]. Additionally, the absolute Seebeck coefficient remained at a high value (around 140-160 $\mu \mathrm{V} / \mathrm{K}$ ) across the whole measured temperature range, even though it decreased slightly because of the increased carrier concentration. Thus, a large power factor of $120 \mu \mathrm{W} / \mathrm{mK}^{2}$ was achieved at $473 \mathrm{~K}$ by annealing the thin SnSe film for $4 \mathrm{~h}$ owing to the combination of an enhanced electrical conductivity and a moderate Seebeck coefficient. This power factor is nearly six times higher than that of n-type bulk polycrystalline $\mathrm{SnSe}_{0.8}$ at the same temperature [22]. Usually, the thermal conductivity of thin films is lower than that of the corresponding bulk material because of the increased scattering introduced by boundaries. Therefore, a higher $Z T$ value than that reported for n-type bulk polycrystalline samples would be obtained in this work via a larger power factor and a lower thermal conductivity. This suggests that using low dimensional films is actually a promising way of achieving good properties in n-type SnSe. Moreover, better TE properties should be achievable 
if the electrical conductivity were further increased by doping the films. In fact, the electrical transport property in the present work is much lower than that of the SnSe single crystal with $\mathrm{Na}$ doping because of the lower electrical conductivity and Seebeck coefficient [12]. Since Na doping can lower the Fermi level and increase the number of carrier pockets, therefore, the TE properties of the film can be optimizing by $\mathrm{Na}$ doping. Furthermore, compared with the SnSe thin films by pulsed laser deposition $[10,27]$, the present results are comparable to those of the film prepared in normal angle deposition, while lower than that prepared in glancing angle deposition. It is reported that the glancing angle deposition can greatly reduce the grain size of the thin film and improve the Seebeck coefficient and power factor [27]. Thus, combining with doping and regulating the microstructure, better TE properties should be realizable.

\section{Conclusions}

A series of n-type $\mathrm{SnSe}$ thin films were fabricated via thermal evaporation using a powder that was directly prepared by mechanical alloying. The results indicate that the crystallinity of the film is gradually enhanced when the current used in a thermal evaporator to supply the evaporation crucible with power is increased from 100 to 130

A. The evaporation of $\mathrm{Se}$ is sensitive to the current applied to heat the crucible, leading to the deviation from the ideal 1:1 ratio for $\mathrm{Sn}$ to Se. The TE performance shows a weak dependence on the thermal evaporator current. However, after annealing the films, their electrical transport properties improved significantly. The combination of the high Seebeck coefficient of $209 \mu \mathrm{V} / \mathrm{K}$ together with the enhanced electrical conductivity of over $5 \mathrm{~S} / \mathrm{cm}$ results in a maximum power factor of 21 $\mu \mathrm{W} / \mathrm{mK}^{2}$ for an n-type conductive film prepared with a thermal evaporation current of $130 \mathrm{~A}$ and an annealing temperature of $673 \mathrm{~K}$. Additionally, the electrical conductivity was further improved to $47 \mathrm{~S} / \mathrm{cm}$ by increasing the annealing time. Thus, an even higher power factor of $120 \mu \mathrm{W} / \mathrm{mK}^{2}$ was achieved when the annealing time was increased to $4 \mathrm{~h}$, indicating that annealing is an efficient way to improve the TE properties of SnSe films. 


\section{Acknowledgements}

Wen-ting Wang and Zhuang-hao Zheng contributed equally. This work was supported by the National Nature Science Foundation (Grant No. 51302140), the Shenzhen Science and Technology Plan Project (Nos. JCYJ20170818142740568, JCYJ20160422102622085 and JCYJ20150827165038323), and by the Natural Science Foundation of SZU (No. 2016016). We thank Jim Bailey, PhD, from Liwen Bianji, Edanz Editing China (www.liwenbianji.cn/ac), for editing the English text of a draft of this manuscript. 


\section{References}

[1] L.E. Bell, Cooling, heating, generating power, and recovering waste heat with thermoelectric systems, Science 321 (2008) 1457-1461.

[2] A.M. Dehkordi, M. Zebarjadi, J. He, T.M. Tritt, Thermoelectric power factor: Enhancement mechanisms and strategies for higher performance thermoelectric materials, Mater. Sci. Eng. R. 97 (2015) 1-22.

[3] F. Li, Z.H. Zheng, Y.W. Li, W.T. Wang, J.F. Li, B. Li, A.H. Zhong, J.T. Luo, P. Fan, Ag-doped $\mathrm{SnSe}_{2}$ as a promising mid-temperature thermoelectric material, J. Mater. Sci. 52 (2017) 10506-10516.

[4] Z.K. Cai, P. Fan, Z.H. Zheng, P.J. Liu, T.B. Chen, X.M. Cai, J.T. Luo, G.X. Liang, D.P. Zhang, Thermoelectric properties and micro-structure characteristics of annealed N-type bismuth telluride thin film, Appl. Surf. Sci. 280 (2013) 225-228.

[5] A. Giani, A. Boulouz, F.P. Delannoy, A. Foucaran, E. Charles, A. Boyer, Growth of $\mathrm{Bi}_{2} \mathrm{Te}_{3}$ and $\mathrm{Sb}_{2} \mathrm{Te}_{3}$ thin films by MOCVD, Mater. Sci. Eng. B 64 (1999) 19-24.

[6] T. Nishino, T. Suzuki, Flexible thermoelectric generator with efficient vertical to lateral heat path films, J. Micromech. Microeng. 27 (2017) 035011-035019.

[7] A. Yadav, K.P. Pipe, M. Shtein, Fiber-based flexible thermoelectric power generator, J. Power Sources 175 (2008) 909-913.

[8] M. Bicer, I. Sisman, Electrodeposition and growth mechanism of SnSe thin films, Appl. Surf. Sci. 257 (2011) 2944-2949.

[9] V.R. Minnam Reddy, S. Gedi, B. Pejjai, C. Park, Perspectives on SnSe-based thin film solar cells: a comprehensive review, J. Mater. Sci. 27 (2016) 5491-5508.

[10] T. Inoue, H. Hiramatsu, H. Hosono, T. Kamiya, Heteroepitaxial growth of SnSe films by pulsed laser deposition using Se-rich targets, J. Appl. Phys. 118 (2015) 205302.

[11] L.D. Zhao, S.H. Lo, Y. Zhang, H. Sun, G. Tan, C. Uher, C. Wolverton, V.P. Dravid, M.G. Kanatzidis, Ultralow thermal conductivity and high thermoelectric figure of merit in SnSe crystals, Nature 508 (2014) 373-377.

[12] K.L. Peng, X. Lu, H. Zhan, S. Hui, X.D. Tang, G.W. Wang, J.Y. Dai, C. Uher, G. Y. Wang, X.Y. Zhou, Broad temperature plateau for high ZTs in heavily doped 
p-type SnSe single crystals, Energy \& Environ. Sci. 9 (2016) 454-460.

[13] G.D. Tang, W. Wei, J. Zhang, Y.S. Li, X. Wang, G.Z. Xu, C. Chang, Z.H. Wang, Y.W. Du, L.D. Zhao, Realizing high figure of merit in phase-separated polycrystalline $\mathrm{Sn}_{1-\mathrm{x}} \mathrm{Pb}_{\mathrm{x}} \mathrm{Se}$, J. Am. Chem. Soc. 138 (41) (2016) 13647-13654.

[14] L.D. Zhao, C. Chang, G.J. Tan, M.G. Kanatzidis, SnSe: a remarkable new thermoelectric material, Energy Environ. Sci. 9 (2016) 3044-3060.

[15] Q. Zhang, E.K. Chere, J.Y. Sun, F. Cao, K. Dahal, S. Chen, G. Chen, Z.F. Ren, Studies on thermoelectric properties of n-type polycrystalline $\mathrm{SnSe}_{1-\mathrm{x}} \mathrm{S}_{\mathrm{x}}$ by iodine doping, Adv. Energy. Mater. 5 (2015) 1500360-1500368.

[16] F. Li, J.F. Li, L.D. Zhao, K. Xiang, Y. Liu, B.P. Zhang, Y.H. Lin, C.W. Nan, H.M. Zhu, Polycrystalline BiCuSeO oxide as a potential thermoelectric material, Energy Environ. Sci. 5 (2012) 7188-7195.

[17] Y. Pan, J.F. Li, Thermoelectric performance enhancement in n-type $\mathrm{Bi}_{2}(\mathrm{TeSe})_{3}$ alloys owing to nanoscale inhomogeneity combined with a spark plasma-textured microstructure, NPG Asia Mater. 8 (2016) e275-e283.

[18] R. Indirajith, T.P. Srinivasan, K. Ramamurthi, R. Gopalakrishnan, Synthesis, deposition and characterization of tin selenide thin films by evaporation technique, Curr. Appl. Phys. 10 (2010) 1402-1406.

[19] D.B. Li, X.J. Tan, J.T. Xu, G.Q. Liu, M. Jin, H.Z. Shao, H.J. Huang, J.F. Zhang, J. Jiang, Enhanced thermoelectric performance in n-type polycrystalline $\mathrm{SnSe}$ by $\mathrm{PbBr}_{2}$ doping, RSC Adv. 7 (2017) 17906-17912.

[20] C.L. Chen, H. Wang, Y.Y. Chen, T. Day, G. J. Snyder, Thermoelectric properties of p-type polycrystalline SnSe doped with Ag, J. Mater. Chem. A 2 (2014) 11171-11176.

[21] B. Zhang, K.L. Peng, A. Li, X.Y. Zhou, Y.H. Chen, Q.S. Deng, X.D. Han, The chemistry and structural thermal stability of hole-doped single crystalline $\mathrm{SnSe}$, J. Alloys Compd. 688 (2016) 1088-1094.

[22] S. Lv, Z.H. Ge, Y.X. Chen, K.Y. Zhao, J. Feng, J.Q. He, Thermoelectric properties of polycrystalline $\mathrm{SnSe}_{1-\mathrm{x}}$ prepared by mechanical alloying and spark plasma sintering, RSC Adv. 6 (2016) 92335-92340. 
[23] D.H. Kim, G.H. Lee, Effect of rapid thermal annealing on thermoelectric properties of bismuth telluride films grown by co-sputtering, Mater. Sci. Eng. B 131 (2006) 106-110.

[24] Y.D. Li, P. Fan, Z.H. Zheng, J.T. Luo, G.X. Liang, S.Z. Guo, The influence of heat treatment on the thermoelectric properties of copper selenide thin films prepared by ion beam sputtering deposition, J. Alloys Compd. 658 (2016) 880-884.

[25] H. Huang, W.L. Luan, S.T. Tu, Influence of annealing on thermoelectric properties of bismuth telluride films grown via radio frequency magnetron sputtering, Thin Solid Films 517 (2009) 3731-3734.

[26] E. Cappelli, A. Bellucci, L. Medici, A. Mezzi, S. Kaciulis, F. Fumagalli, F.D. Fonzo, D.M. Trucchi, Nano-crystalline Ag-PbTe thermoelectric thin films by a multi-target PLD system, Appl. Surf. Sci. 336 (2015) 283-289.

[27] C.H. Suen, D.L. Shi, Y. Su, Z. Zhang, C.H. Chan, X.D. Tang, Y. Li, K.H. Lam, X.X. Chen, B.L. Huang, X.Y. Zhou, J.Y. Dai, Enhanced thermoelectric properties of SnSe thin films grown by pulsed glancing-angle deposition, J. Materiomics 3(2017) 293-298. 


\section{Figure captions}

Fig. 1. XRD patterns of the as-deposited films obtained at different crucible currents during the thermal evaporation of the powders.

Fig. 2. SEM images of the as-deposited films obtained at different crucible currents during the thermal evaporation of the powders, namely at (a) $100 \mathrm{~A}$, (b) $110 \mathrm{~A}$, (c) $120 \mathrm{~A}$, and (d) $130 \mathrm{~A}$.

Fig. 3. Electrical transport properties as a function of temperature for the films obtained at different thermal evaporation current intensities. Panel (a) shows the electrical conductivities, panels (b) shows the Seebeck coefficients, and panel (c) shows the power factors.

Fig. 4. XRD patterns of the as-deposited films after heat treatment at different temperatures.

Fig. 5. SEM images of the as-deposited films after heat treatment at different temperatures, namely at (a) $473 \mathrm{~K}$, (b) $573 \mathrm{~K}$, and (c) $673 \mathrm{~K}$.

Fig. 6. Electrical transport properties as a function of temperature for the SnSe thin films annealed at different temperatures. Panel (a) shows the electrical conductivities, panel (b) shows the Seebeck coefficients, and panel (c) shows the power factors.

Fig. 7. Electrical transport properties as a function of temperature for the films after annealing for either 1 or $4 \mathrm{~h}$. Panel (a) shows the electrical conductivities and the Seebeck coefficients, and panel (b) shows the power factors.

Table 1 The composition of $\mathrm{Sn}$ to $\mathrm{Se}$ of the as-deposited films obtained under different thermal evaporation current as detected via EDS.

\begin{tabular}{cccc}
\hline Current intensity (A) & Sn (at\%) & Se (at\%) & Sn/Se ratio \\
\hline $\mathbf{1 0 0}$ & 99.4 & 0.6 & 165.5 \\
$\mathbf{1 1 0}$ & 71.7 & 28.3 & 2.5 \\
$\mathbf{1 2 0}$ & 62.7 & 37.3 & 1.7 \\
$\mathbf{1 3 0}$ & 57.2 & 42.8 & 1.3 \\
\hline
\end{tabular}




\section{Figures}

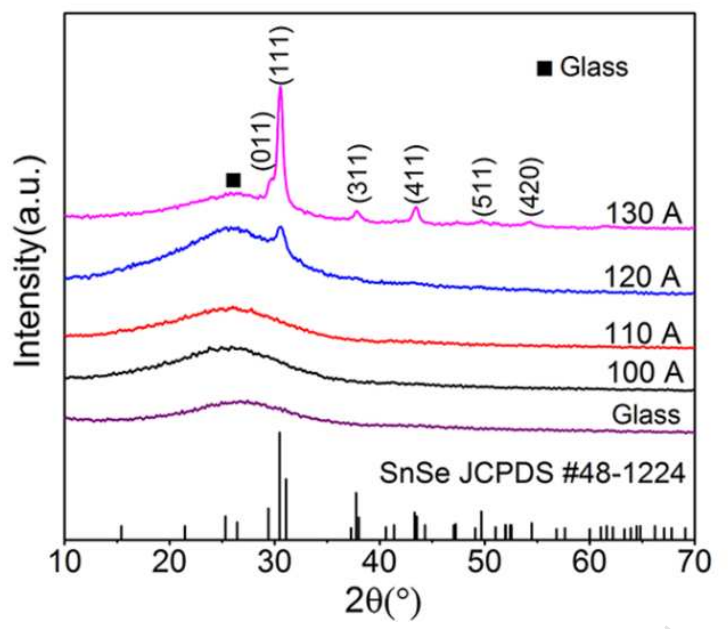

Figure 1

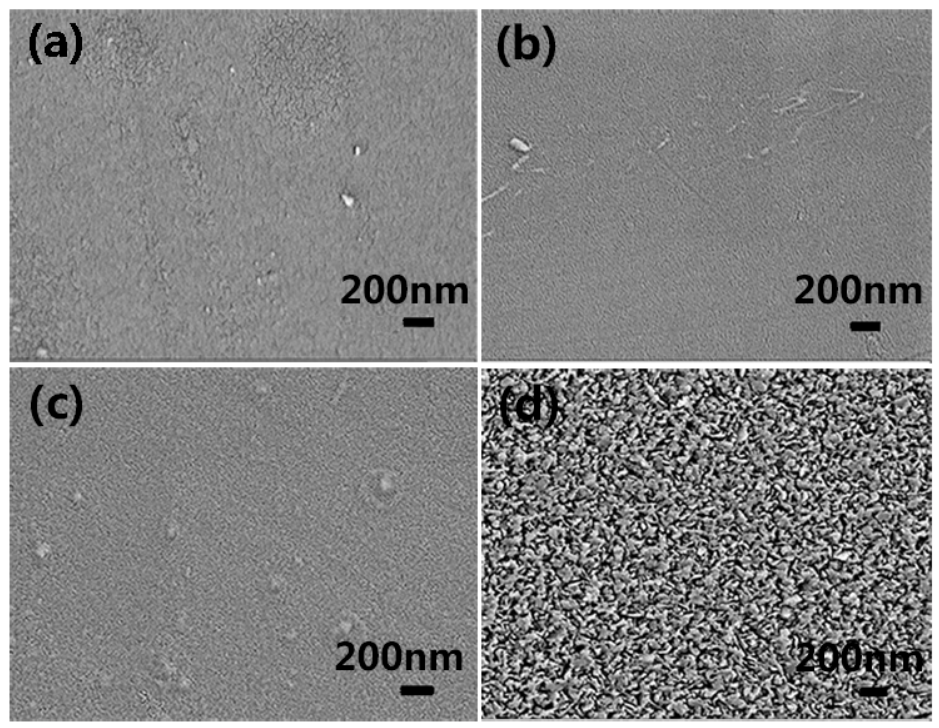

Figure 2 

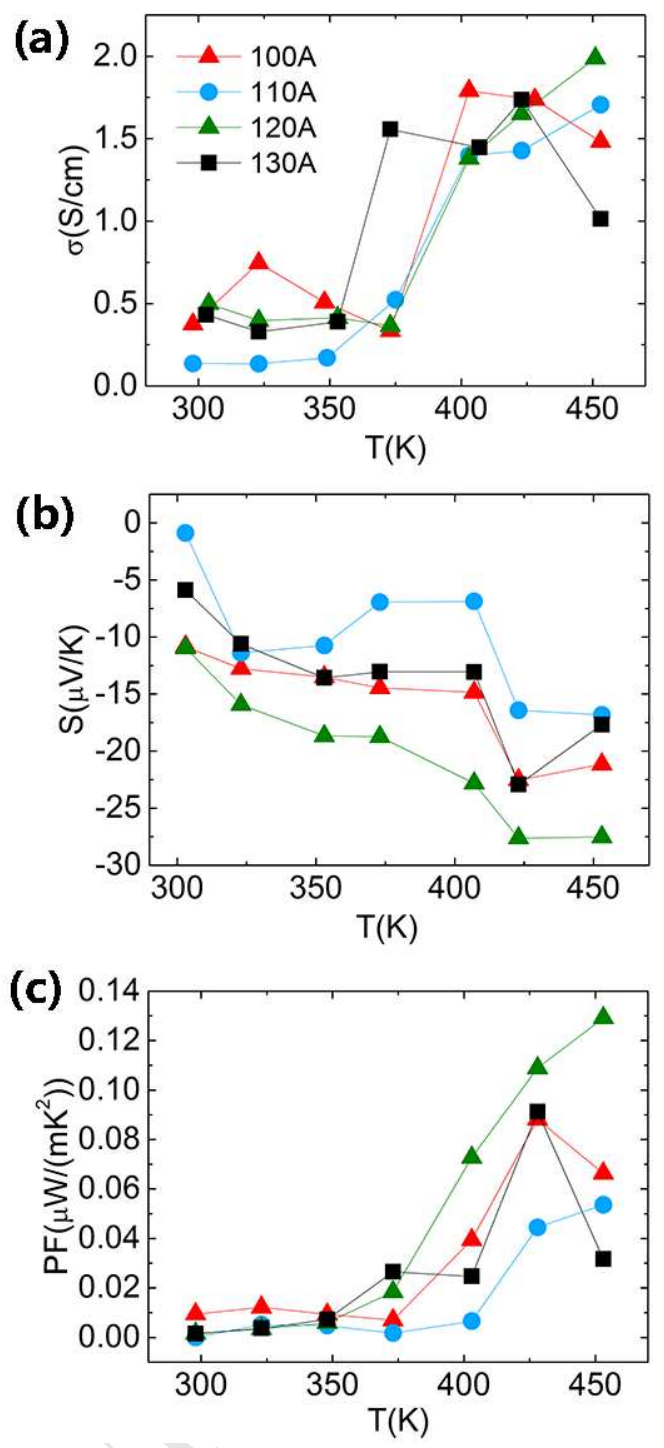

Figure 3 


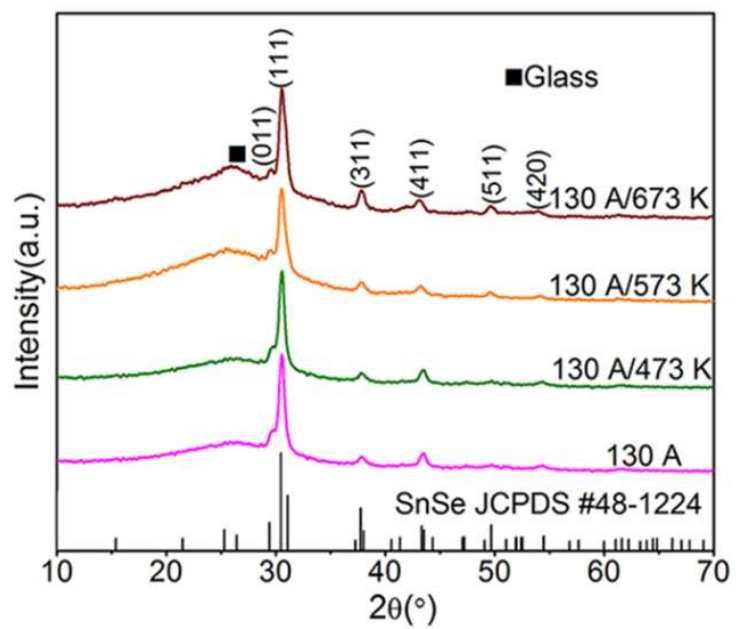

Figure 4

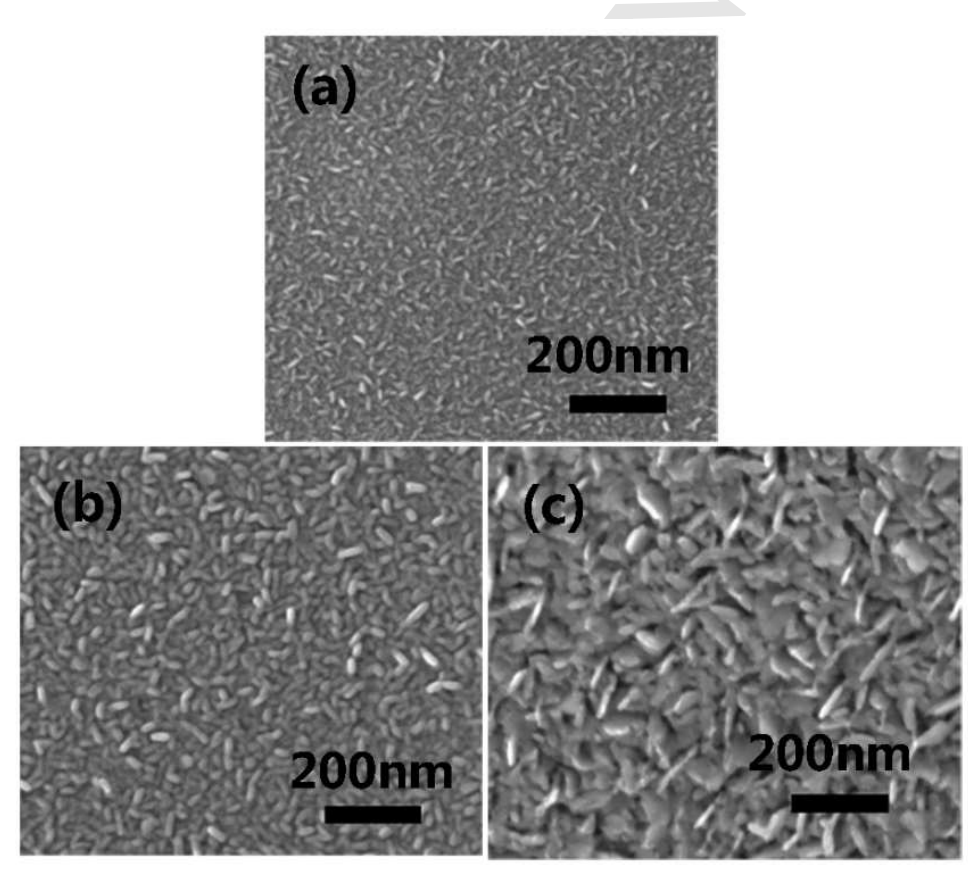

Figure 5 
(a)
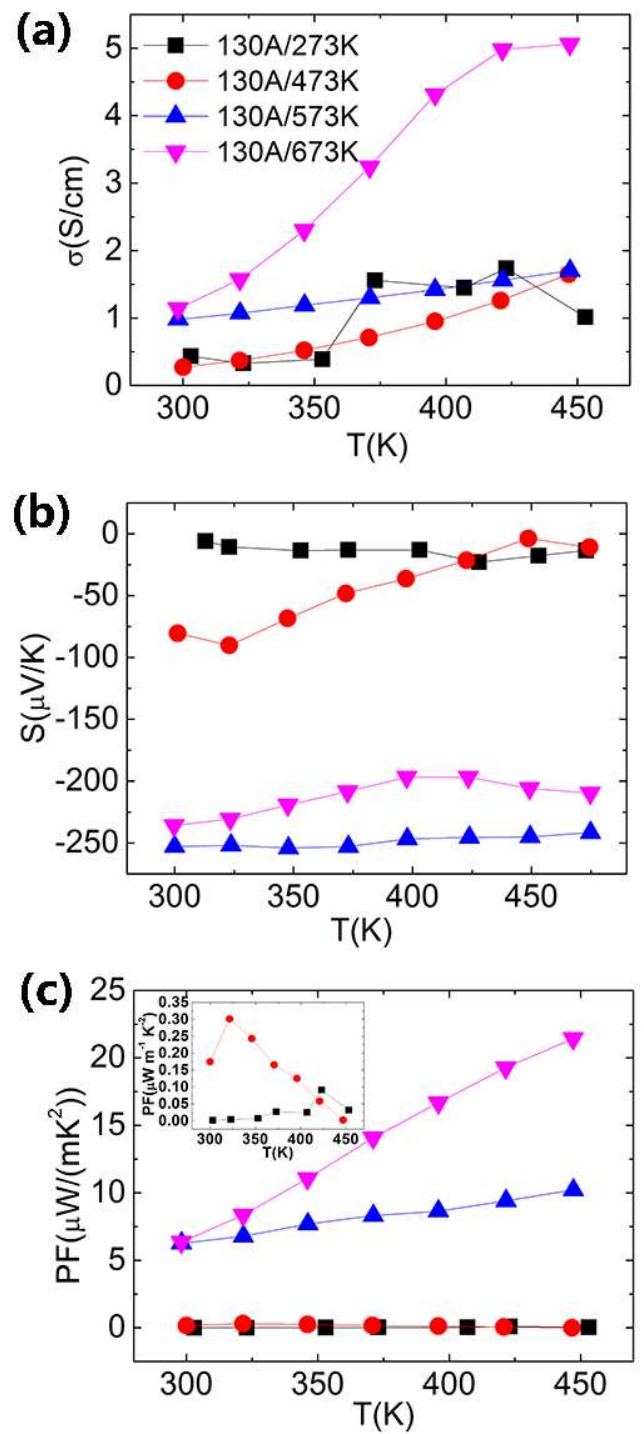

Figure 6 
(a)

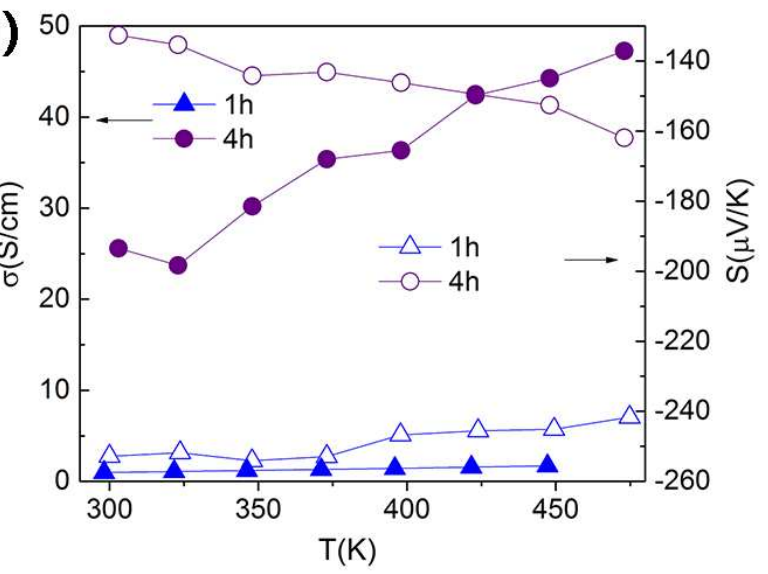

(b)

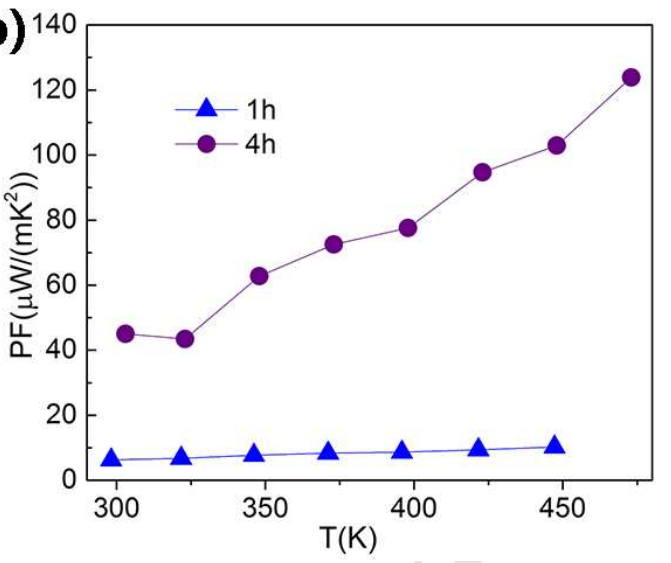

Figure 7 


\section{Highlights}

1. N-type tin selenide thin films were synthesized by thermal evaporation.

2. The electrical transport properties of the films after annealing have greatly improved.

3. A maximum power factor of $120 \mu \mathrm{W} / \mathrm{mK}^{2}$ was achieved at $473 \mathrm{~K}$ for the annealed film. 